\title{
Quality of Life is Diminished in Patients with Tetralogy of Fallot with Mild Residual Disease: A Comparison of Tetralogy of Fallot and Isolated Valvar Pulmonary Stenosis
}

\author{
Shivani M. Bhatt ${ }^{1}$, Elizabeth Goldmuntz ${ }^{1}$, Amy Cassedy ${ }^{2}$, Bradley S. Marino ${ }^{3}$, and Laura \\ Mercer-Rosa ${ }^{1}$ \\ 1'Division of Cardiology, The Children's Hospital of Philadelphia, 3400 Civic Center Boulevard, \\ Philadelphia, PA 19104, USA \\ ${ }^{2}$ Cincinnati Children's Hospital Medical Center, Cincinnati, OH, USA \\ ${ }^{3}$ Ann \& Robert H. Lurie Children's Hospital of Chicago, Chicago, IL, USA
}

\section{Abstract}

The objective of this study is to compare quality of life (QOL) in patients with mild pulmonary insufficiency (PI) after Tetralogy of Fallot (TOF) repair or after balloon dilation for isolated valvar pulmonary stenosis (VPS). A cross-sectional study of patients with TOF $(n=12)$ and VPS $(n=$ $19)$, ages $8-18$ years, who underwent cardiac magnetic resonance (CMR) and cardiopulmonary exercise test (CPET) was conducted. Patients with genetic syndromes were excluded. The groups were matched by severity and duration of PI using propensity scores. PI was greater than mild if the regurgitant fraction by CMR was $>20 \%$. Health status and QOL assessment included Child Health Questionnaire Child Self-Report (CHQ-CF87), Child Health Questionnaire Parent Report (CHQ-PF50), and Pediatric Cardiac QOL Instrument (parent and patient). QOL scores were compared between groups. Due to propensity matching, the groups had, at worst, mild PI and normal right ventricular ejection fraction on CMR. Parental perception of QOL was significantly worse in TOF as compared to VPS in the domains of general perception of health $(P=0.03)$, physical functioning (PF; $P=0.004)$, and family cohesion $(P=0.048)$. There were no differences in self-reported QOL between groups. There was no association between QOL and right ventricular function on CMR or percent-predicted maximal oxygen consumption on CPET in both groups. Parent-perceived QOL, in the domains of general health perception, PF, and family cohesion, is significantly reduced in patients with TOF with mild residual disease suggesting that the psychosocial impact of congenital heart disease may be significant even with successful repair and satisfactory medical status.

Correspondence to: Shivani M. Bhatt.

Compliance with Ethical Standards

Research Involving Human Participants and/or Animals All procedures performed in studies involving human participants were in accordance with the Ethical Standards of the Institutional and/or National Research Committee and with the 1964 Helsinki Declaration and its later amendments or comparable ethical standards.

Informed Consent Informed consent was obtained from all individual participants included in the study.

Conflict of interest The authors declare that they have no conflict of interest. 


\section{Keywords}

Congenital heart disease; Health status; Quality of life; Pulmonary stenosis; Tetralogy of Fallot

\section{Introduction}

Although long-term survival after surgical repair for Tetralogy of Fallot (TOF) is comparable to the general population, patients experience significant morbidity primarily associated with residual pulmonary insufficiency (PI). In the long term, PI results in right ventricular dilation and ultimately in right ventricular dysfunction with a resultant progressive decline in exercise tolerance [1-4]. These morbidities result in repeated procedures and re-interventions to preserve right ventricular function. Patients also face challenges with cognitive function, education, and employment as adults [5, 6]. Psychosocial morbidities [7-9], neurodevelopmental morbidities [10, 11], and diminished physical functioning (PF) [12] can have a negative cumulative effect on quality of life (QOL) [13]. As an important multidimensional measure of well-being, QOL reflects an individual's ability to function in social, emotional, psychological, and physical domains as well as personal satisfaction [13-15]. QOL has been shown to be diminished in patients with CHD as compared to the general population [16-18].

Our objective was to evaluate the health status and QOL of patients with mild residual disease, defined as mild PI with preserved ejection fraction. We therefore sought to investigate health status and QOL in children and adolescents with repaired TOF as compared to patients with valvar pulmonary stenosis (VPS) status post balloon dilation matched for age and residual disease burden. We hypothesized that health status and QOL would be comparable in these groups.

\section{Methods}

\section{Study Population and Data Collection}

We conducted a single-center cross-sectional study of subjects with VPS ages 8-18 years at The Children's Hospital of Philadelphia (CHOP) from June 2009 to February 2010 as previously described [19]. This study was approved by the Institutional Review Board for the Protection of Human Subjects at CHOP (IRB \#6372). All subjects provided written informed consent prior to participation. VPS subjects status post pulmonary valve balloon dilation identified from the cardiac databases at our institution underwent one day of testing that included a protocol-based cardiac magnetic resonance (CMR), cardiopulmonary exercise test (CPET), and completion of health status and QOL questionnaires by patients and their parent-proxy reporters. Patient health status was measured using the Child Health Questionnaire Child Self-Report (CHQ-CF87) and the Child Health Questionnaire Parent Report (CHQ-PF50), while QOL assessment included a disease-specific instrument, the Pediatric Cardiac QOL Instrument (PCQLI) [20]. The VPS cohort was found to have mild residual disease, defined as mild residual PI after balloon dilation with preserved ventricular ejection fraction. 
TOF subjects were drawn from a cohort with repaired TOF enrolled in a cross-sectional study between January 2005 and February 2009, for which they underwent the same CMR, CPET, and QOL protocol [21]. Testing was performed within 3 months in the TOF group. Patients with genetic syndromes were excluded from this analysis. Subjects with VPS and TOF were matched by duration and severity of PI [years from initial procedure and pulmonary regurgitant fraction (RF) on CMR, respectively] using propensity score matching. This resulted in a group of TOF subjects with mild disease, given that the VPS group had on average mild residual PI after balloon dilation [19, 22]. In this study, the health status of the TOF group and VPS group was compared with healthy control population using published normative data available for the CHQ-PF50 [23, 24]. QOL in the VPS and TOF groups was compared with two CHD control groups based on the severity of disease using published data for mild [isolated bicuspid aortic valve (BAV) with no aortic stenosis or insufficiency who have not undergone intervention] and severe CHD (patients with single ventricle who have undergone Fontan operation) [20, 25].

\section{Health Status and Quality of Life Instruments}

The CHQ is a comprehensive self-report instrument that measures psychosocial and physical well-being which has been validated for the general population of children ages 5-18 years by the Health Institute at New England Medical Center [23]. This tool provides qualitative assessment of overall health status across multiple domains. The Child Health Questionnaire Parent Form 50 (CHQ-PF50) comprises 50 items and is completed by a parent-proxy reporter. Overall well-being is measured by psychosocial (PsS) and physical $(\mathrm{PhS})$ health summary scores and 12 individual health concepts are assessed by subscale scores, including PF, bodily pain/discomfort, role/social limitations_-both physical and emotional/behavioral, self-esteem, mental health, behavior, general health perceptions, family activities, family cohesion, and parental impact — both time and emotional. The $\mathrm{PhS}$ is generated from the weighted average of subscale scores of PF, role/social-physical, general health, and bodily pain. The PsS is derived from the subscale scores of self-esteem, mental health, behavior, and role/social-emotional behavioral. Both summary measures include subscales evaluating time and emotional impact on the parent. A higher score reflects better overall physical and mental health status. The Child Health Questionnaire Child Form 87 (CHQ-CF87) is completed by the child and contains 87 questions assessing the health concepts corresponding to the CHQ-PF50 subscales with the exception of the parental impact subscales and distinction of the role/social-emotional and role/social-behavioral subscales. Summary scores are not generated [23].

\section{PCQLI}

The Pediatric Cardiac QOL Inventory (PCQLI) is a reliable and validated instrument of disease-specific QOL in pediatric heart disease [13, 15, 20]. The PCQLI has both child and parent-proxy forms: child (8-12 years), parent of child (parent-proxy), adolescent (13-18 years), and parent of adolescent (parent-proxy) forms. Disease impact and psychosocial impact subscale scores are generated with a maximum of 50 points each. A total PCQLI score is generated from the sum of subscale scores and higher scores represent better healthrelated QOL [20]. Significant differences in PCQLI QOL scores have been demonstrated between patients with mild CHD not requiring surgical intervention, moderate CHD with 
biventricular physiology, and severe CHD (patients with single-ventricle physiology after Fontan operation) [20, 25].

Cardiac Magnetic Resonance-CMR studies were performed using a standard imaging protocol and read by an experienced physician blinded to the patients' clinical information, as previously described [19]. PI was measured as pulmonary RF and considered mild if it is $<20 \%$, moderate if $21-40 \%$, and severe if greater than $40 \%$. Other CMR variables included indexed end-systolic and end-diastolic RV volumes and RV ejection fraction. RV ejection fraction was considered normal if it is greater than $50 \%$ [26, 27].

Cardiopulmonary Exercise Test-Patients completed an exercise protocol as previously described [19]. The percent of predicted maximum $V_{\mathrm{O}_{2}}\left(\% \mathrm{mV}_{\mathrm{O}_{2}}\right)$ was calculated for each patient, according to normative values for age, gender, and body size. Percentpredicted $\mathrm{mV}_{\mathrm{O}_{2}}$ was considered abnormal if less than $85 \%$ predicted [19]. Oxygen pulse was calculated as the $\mathrm{mV}_{\mathrm{O}_{2}}$ divided by the peak heart rate and indexed for body surface area $\left(\mathrm{ml} / \mathrm{O}_{2} /\right.$ beat $\left./ \mathrm{m}^{2}\right)$ and used as a surrogate of stroke volume.

\section{Statistical Analysis}

The groups were matched by RF and duration of PI using propensity scores. Continuous variables are presented as median with interquartile ranges (IQRs) or as mean and standard deviation (SD) as appropriate. Categorical variables are described using frequencies and percentages. The Mann-Whitney $U$ test for continuous variables and $\chi^{2}$ test for categorical variables were performed to compare health status and QOL between the VPS and TOF groups. Linear regression was used to examine the relationship between the outcome variables, QOL and $\mathrm{mV}_{\mathrm{O}_{2}}$. Normative data for the CHQ-PF50 were used to compare VPS and TOF subscale scores with published norms via paired sample $t$ tests. In addition, paired sample $t$ tests were used to compare PCQLI scores of the VPS and TOF groups with two CHD control groups: mild CHD (isolated BAV without history of intervention) and severe CHD (single ventricle who have undergone Fontan palliation) [18, 25]. An $a$ of $<0.05$ was considered significant and all analyses were conducted using SAS@9.2.

\section{Results \\ Patient Characteristics}

Matching for severity and duration of PI identified 12 subjects with TOF and 19 with VPS. The groups were comparable in age, race, and gender (Table 1). By study design, subjects had comparable PI, measured as RF. There was mild residual PI and less than mild RV dilation with preserved right ventricular function in both groups. Both the percent-predicted maximal oxygen consumption and the oxygen pulse were comparable in the groups (Table 1). All TOF patients underwent cardiac surgical repair and required at least one catheterization procedure (Table 2). VPS patients did not require cardiac surgery and underwent transcatheter pulmonary valve balloon dilation. 


\section{Health Status and QOL Comparisons to Normative Populations}

VPS Group Comparisons to Normative Sample-In the VPS group, the Parent

(CHQ-PF50) health status scores were comparable to a normative sample, as there were no significant differences in summary or subscale scores (Table 3). VPS patients had statistically lower psychosocial impact and total PCQLI scores than patients with mild CHD. There was no difference in patient disease impact scores between the VPS and mild CHD groups. Parent-proxy psychosocial score was also significantly lower in the VPS compared to the mild CHD group, with no difference in parent-proxy disease impact or total scores (Table 4). Patients in the VPS group had statistically higher patient disease impact scores than those with severe CHD. There was no difference in patient psychosocial impact or total scores. The parent-proxy disease impact scores and parent-proxy total scores in the VPS group were also significantly better than those in the severe CHD group, with no difference in parent-proxy psychosocial impact scores (Table 5).

TOF Group Comparisons to Normative Sample-In the TOF group, the Parent (CHQ-PF50) physical and psychosocial summary scores were comparable to the normative healthy sample. However, lower scores in several subscales were apparent including general health perception $(P=0.0001)$ and family cohesion $(P=0.035$; Table 3$)$. TOF patients had better bodily pain scores compared to the normative sample. TOF patients had PCQLI psychosocial impact scores that were significantly lower than patients with mild CHD. There was no difference in patient disease impact or total PCQLI scores between TOF and mild CHD patients. Parent-proxy psychosocial impact and total PCQLI scores were also lower in TOF compared with patients with mild CHD. There was no difference in parent-proxy disease impact scores (Table 4). Compared with severe CHD patients, TOF patients had significantly better patient and parent-proxy disease impact scores. No difference was found in patient psychosocial impact or total scores between TOF and severe CHD patients. Parent-proxy psychosocial impact scores, however, were significantly lower in TOF patients compared with severe CHD patients. There was no difference in total parent-proxy scores between TOF and severe CHD groups (Table 5).

\section{Comparison of VPS with TOF Groups}

Parent-Proxy Health Status (CHQ-PF50)—Although parent-proxy physical and psychosocial summary scores were similar among patients with TOF and VPS, certain subscales were significantly lower in TOF as compared to VPS, including parent-proxyreported general health perception, PF, and family cohesion. Subscale scores in the major psychosocial domains including global behavior, global health, behavior, mental health, selfesteem, social limitations (behavioral and physical), parental impact, and family factors were comparable between the groups (Table 6).

Child Health Status (CHQ-CF87) - Self-reported mental health scores were better in patients with TOF compared to VPS. All other self-reported QOL scores were comparable between the groups (Table 7).

Parent-Proxy and Child QOL (PCQLI)-Parents of patients in the TOF group reported similar overall QOL as those in the VPS group, as evident by the parent-proxy PCQLI total 
score. There were no group differences in parent-reported PCQLI disease impact or psychosocial impact scores. There were no significant group differences in any of the patient-reported PCQLI measures of QOL (Table 7).

\section{Discussion}

In this study, we sought to investigate the differences in health status and QOL in children and adolescents with TOF and VPS with mild residual disease, defined as mild PI with preserved ejection fraction. In comparison to patients with VPS matched for similar residual disease burden, parent-proxy-reported QOL scores in the domains of general health perception, $\mathrm{PF}$, and family cohesion were significantly reduced in patients with repaired TOF. In TOF with mild residual disease, parent-proxy-reported QOL scores in the domains of general health perceptions and family cohesion were lower compared to healthy children. Furthermore, parental psychosocial QOL was significantly lower in TOF than mild and severe CHD groups. Although parent-proxy-reported health status in VPS with mild residual disease was comparable to healthy children, their psychosocial QOL scores were significantly lower in VPS compared to those with mild CHD.

Even in the face of mild residual disease, parent-proxy-reported general health perception was significantly lower in TOF as compared to VPS. Furthermore, parent-proxy psychosocial impact was lower in TOF than those with mild and severe CHD. This suggests that in TOF, the parent-perceived QOL is lower than the severity of residual disease. Alternatively, our finding might suggest that having a child that underwent cardiac surgery might, in and of itself, result in impaired QOL when compared to parents of children that underwent nonsurgical procedures (such as balloon dilation for VPS). Similarly to our results, Mellion et al. demonstrated that parent-reported QOL scores in adolescents with mild CHD (such as VPS) were higher than in those with other biventricular CHD such as repaired TOF [18]. Other studies in TOF have demonstrated that parents perceive their children to have diminished self-esteem and general health [10]. In addition, the parentreported QOL is diminished in children and adolescents with TOF and comparable to chronically ill children [28]. Contrary to our findings, Uzark et al. demonstrated that parentperceived QOL in children and adolescents with CHD was similar to healthy children; however, the study found an incremental decrease in parent-perceived QOL with increasing severity of CHD [29].

Parent-reported PF was worse in the TOF group as compared to VPS. Other studies have also shown that adults with repaired TOF report worse physical health status despite selfreported QOL that is similar to healthy peers [30,31]. Diminished PF has also been shown in adolescents with repaired TOF; however, worse PF was associated with RV systolic dysfunction as well as with concurrent symptoms and number of operations [10, 32]. We found worse parent-reported PF in TOF patients despite preserved right ventricular function, and while the aerobic capacity was low in the TOF group, it was not associated with reported PF. These findings suggest that factors aside from clinical status may play a role in parent-reported PF. One possible explanation is that parents of children with CHD generally underestimate their child's exercise capacity [33]. We did not find an association between 
reported PF and aerobic capacity on CPET in this study, even though abnormal oxygen consumption was apparent in the TOF group.

The assessment of parental perception of their child's QOL is important as health care utilization is typically associated with parent-proxy QOL [34]. Familial factors beyond the degree of heart disease may drive parental perceptions of QOL. Adverse family relationships have been shown to negatively impact psychosocial QOL [17], whereas positive family characteristics, such as high family cohesion, increased expressiveness, and less intrafamilial conflict, have been shown to favorably influence parent-proxy QOL in CHD [17]. We found that the parent-proxy-reported family cohesion score was significantly worse in the TOF compared to the VPS group. Parental well-being and ability to cope with their child's heart disease play a significant role in family cohesion, where worse family cohesion is associated with higher parental psychological distress [35]. Our findings suggest that interventions to strengthen family welfare and family support that integrate both the child's individual needs as well as parental needs may improve psychosocial QOL.

Parental stress is also associated with QOL and higher levels of parental psychological distress have been associated with more severe heart defects at primary diagnosis [36, 37]. The initial diagnosis is a stressful time for parents of children with CHD and diagnosis is increasingly more common in the prenatal period $[38,39]$. During prenatal counseling, parents of children with CHD place a greater importance on QOL of their future child, including the number of lifetime interventions and physical limitations than their fetal cardiologist [40]. Parental perceptions of their child's CHD and anticipated QOL formed during prenatal counseling may influence their expectations, psychological preparation, and coping with their child's illness. It is possible that despite the mild residual disease after surgical repair in this study group, psychological stress is higher in parents of children with TOF compared to VPS. This may be due to the parental perception of illness severity at initial diagnosis, given that patients with TOF undergo open heart surgery and possibly more future re-interventions than patients with VPS. Parental stress is associated with parental perception of illness and is greater in parents of children with repaired single-ventricle heart disease when compared to those with two-ventricle repairs [7]. Conversely, parental stress can also be unrelated to the severity of $\mathrm{CHD}$, suggesting the need for practitioners to consider measures to address parental stress irrespective of underlying CHD diagnosis [41].

To our knowledge, QOL in VPS patients after balloon dilation has not been previously evaluated. We found that parent-proxy-reported QOL in VPS with mild residual disease after balloon dilation was similar to that in a normative healthy population. However, when compared with a national sample of patients with mild CHD (BAV) who did not require surgical intervention, both parent and patient QOL scores particularly with respect to psychosocial impact were lower. Our findings suggest that although VPS patients are in many cases considered to be on the mild end of the spectrum of CHD, the need for intervention such as balloon dilation might have a detrimental impact on their QOL, particularly from a psychosocial standpoint.

Risk factors aside from underlying disease such as lack of psychosocial support may lead to worse parental psychological distress and poor parent and child well-being [35]. The 
availability of social support and resources to families with a child affected by CHD has been associated with better QOL possibly due to promotion of resilience and improved adaptation in patients with heart disease [42]. Furthermore, parents of children with CHD report worse QOL for themselves than parents of healthy children, and parents' own mental status, coping abilities, and self-perceived QOL may moderate the perception of their child's QOL $[43,44]$.

This study was limited by its small sample size, but significant differences that point to diminished general perception of health, PF, and family cohesion even in the face of mild disease were apparent. In both groups, the QOL data were acquired prospectively as part of research study protocols lending strength to our findings. Furthermore, we used both generic and disease-specific instruments to provide a multidimensional assessment of QOL. Because we matched patients with TOF to those with VPS and mild disease by duration and severity of PI, the resulting groups have mild residual disease as dictated by VPS so they are not generalizable to TOF patients with more severe disease.

\section{Conclusions}

Parent-reported QOL is affected in children with CHD, even in the context of mild residual disease. Furthermore, in certain domains, QOL is worse in TOF patients than in those with VPS in the context of mild residual disease. It is possible that having a relatively more severe disease (TOF) with open heart surgery early in life is the main driver of these differences. Our findings should alert health care providers that QOL might be diminished even in the face of mild residual disease. Strategies to improve family cohesion, manage parental stress, and promote coping may result in improved QOL.

\section{Acknowledgments}

This study was supported by NHLBI HL7473 (Goldmuntz), CHOP Cardiac Center Grant (Mercer-Rosa), NIH U01HL098153 (Goldmuntz), NIH K01HL125521 (Mercer-Rosa), and NIH 5T32HL007915 (Bhatt).

\section{Abbreviations}

$\begin{array}{ll}\text { QOL } & \text { Quality of life } \\ \text { TOF } & \text { Tetralogy of Fallot } \\ \text { VPS } & \text { Isolated valvar pulmonary stenosis } \\ \text { CMR } & \text { Cardiac magnetic resonance } \\ \text { CPET } & \text { Cardiopulmonary exercise test } \\ \text { RF } & \text { Regurgitant fraction } \\ \text { PI } & \text { Pulmonary insufficiency }\end{array}$




\section{References}

1. Apitz C, Webb GD, Redington AN. Tetralogy of Fallot. Lancet. 2009; 374(9699):1462-1471. [PubMed: 19683809]

2. Bacha EA, Scheule AM, Zurakowski D, et al. Long-term results after early primary repair of Tetralogy of Fallot. J Thorac Cardiovasc Surg. 2001; 122(1):154-161. [PubMed: 11436049]

3. Hickey EJ, Veldtman G, Bradley TJ, et al. Late risk of outcomes for adults with repaired Tetralogy of Fallot from an inception cohort spanning four decades. Eur J Cardiothorac Surg. 2009; 35(1): 156-164. discussion 164. [PubMed: 18848456]

4. Marelli AJ, Ionescu-Ittu R, Mackie AS, Guo L, Dendukuri N, Kaouache M. Lifetime prevalence of congenital heart disease in the general population from 2000 to 2010. Circulation. 2014; 130(9): 749-756. [PubMed: 24944314]

5. Wray J, Sensky T. Congenital heart disease and cardiac surgery in childhood: effects on cognitive function and academic ability. Heart. 2001; 85(6):687-691. [PubMed: 11359753]

6. Daliento L, Mapelli D, Russo G, et al. Health related quality of life in adults with repaired Tetralogy of Fallot: psychosocial and cognitive outcomes. Heart. 2005; 91(2):213-218. [PubMed: 15657236]

7. Brosig CL, Mussatto KA, Kuhn EM, Tweddell JS. Psychosocial outcomes for preschool children and families after surgery for complex congenital heart disease. Pediatr Cardiol. 2007; 28(4):255262. [PubMed: 17486393]

8. Casey FA, Sykes DH, Craig BG, Power R, Mulholland HC. Behavioral adjustment of children with surgically palliated complex congenital heart disease. J Pediatr Psychol. 1996; 21(3):335-352. [PubMed: 8935237]

9. Kamphuis M, Ottenkamp J, Vliegen HW, et al. Health related quality of life and health status in adult survivors with previously operated complex congenital heart disease. Heart. 2002; 87(4):356362. [PubMed: 11907011]

10. Neal AE, Stopp C, Wypij D, et al. Predictors of health-related quality of life in adolescents with Tetralogy of Fallot. J Pediatr. 2015; 166(1):132-138. [PubMed: 25444004]

11. Gerstle M, Beebe DW, Drotar D, Cassedy A, Marino BS. Executive functioning and school performance among pediatric survivors of complex congenital heart disease. J Pediatr. 2016; doi: 10.1016/j.jpeds.2016.01.02

12. Pilla CB, Pereira CA, Fin AV, et al. Health-related quality of life and right ventricular function in the midterm follow-up assessment after Tetralogy of fallot repair. Pediatr Cardiol. 2008; 29(2): 409-415. [PubMed: 18026777]

13. Marino BS, Shera D, Wernovsky G, et al. The development of the pediatric cardiac quality of life inventory: a quality of life measure for children and adolescents with heart disease. Qual Life Res. 2008; 17(4):613-626. [PubMed: 18347927]

14. Drotar D. Validating measures of pediatric health status, functional status, and health-related quality of life: key methodological challenges and strategies. Ambul Pediatr. 2004; 4(4 Suppl): 358-364. [PubMed: 15264947]

15. Marino BS, Drotar D, Cassedy A, et al. External validity of the pediatric cardiac quality of life inventory. Qual Life Res. 2011; 20(2):205-214. [PubMed: 21188538]

16. Lane DA, Lip GY, Millane TA. Quality of life in adults with congenital heart disease. Heart. 2002; 88(1):71-75. [PubMed: 12067950]

17. Landolt MA, Valsangiacomo Buechel ER, Latal B. Health-related quality of life in children and adolescents after open-heart surgery. J Pediatr. 2008; 152(3):349-355. [PubMed: 18280839]

18. Mellion K, Uzark K, Cassedy A, et al. Health-related quality of life outcomes in children and adolescents with congenital heart disease. J Pediatr. 2014; 164(4):781-788. e781. [PubMed: 24412135]

19. Mercer-Rosa L, Ingall E, Zhang X, et al. The impact of pulmonary insufficiency on the right ventricle: a comparison of isolated valvar pulmonary stenosis and Tetralogy of Fallot. Pediatr Cardiol. 2015; 36(4):796-801. [PubMed: 25519915]

20. Marino BS, Tomlinson RS, Wernovsky G, et al. Validation of the pediatric cardiac quality of life inventory. Pediatrics. 2010; 126(3):498-508. [PubMed: 20805147] 
21. Mercer-Rosa L, Yang W, Kutty S, Rychik J, Fogel M, Goldmuntz E. Quantifying pulmonary regurgitation and right ventricular function in surgically repaired Tetralogy of Fallot: a comparative analysis of echocardiography and magnetic resonance imaging. Circ Cardiovasc Imaging. 2012; 5(5):637-643. [PubMed: 22869820]

22. Mercer-Rosa L, Paridon SM, Fogel MA, et al. 22q11.2 deletion status and disease burden in children and adolescents with Tetralogy of Fallot. Circ Cardiovasc Genet. 2015; 8(1):74-81. [PubMed: 25561045]

23. Landgraf, JMAL., Ware, JE. The child health questionnaire (CHQ): a user's manual. The Health Institute, New England Medical Center; Boston: 1999.

24. HealthActCHQ. The CHQ scoring and interpretation manual. HealthActCHQ; Boston: 2013.

25. Czosek RJ, Kaltman JR, Cassedy AE, et al. Quality of life of pediatric patients with long QT syndrome. Am J Cardiol. 2016; 117(4):605-610. [PubMed: 26721659]

26. Sarikouch S, Peters B, Gutberlet M, et al. Sex-specific pediatric percentiles for ventricular size and mass as reference values for cardiac MRI: assessment by steady-state free-precession and phasecontrast MRI flow. Circ Cardiovasc Imaging. 2010; 3(1):65-76. [PubMed: 19820203]

27. Robbers-Visser D, Boersma E, Helbing WA. Normal biventricular function, volumes, and mass in children aged 8 to 17 years. J Magn Reson Imaging. 2009; 29(3):552-559. [PubMed: 19243036]

28. Kwon EN, Mussatto K, Simpson PM, Brosig C, Nugent M, Samyn MM. Children and adolescents with repaired Tetralogy of Fallot report quality of life similar to healthy peers. Congenit Heart Dis. 2011; 6(1):18-27. [PubMed: 21269409]

29. Uzark K, Jones K, Slusher J, Limbers CA, Burwinkle TM, Varni JW. Quality of life in children with heart disease as perceived by children and parents. Pediatrics. 2008; 121(5):e1060-e1067. [PubMed: 18450848]

30. Knowles R, Veldtman G, Hickey EJ, et al. Functional health status of adults with Tetralogy of Fallot: matched comparison with healthy siblings. Ann Thorac Surg. 2012; 94(1):124-132. [PubMed: 22429671]

31. Hickey EJ, Veldtman G, Bradley TJ, et al. Functional health status in adult survivors of operative repair of Tetralogy of Fallot. Am J Cardiol. 2012; 109(6):873-880. [PubMed: 22244382]

32. Lu JC, Cotts TB, Agarwal PP, Attili AK, Dorfman AL. Relation of right ventricular dilation, age of repair, and restrictive right ventricular physiology with patient-reported quality of life in adolescents and adults with repaired Tetralogy of Fallot. Am J Cardiol. 2010; 106(12):1798-1802. [PubMed: 21126623]

33. Casey FA, Craig BG, Mulholland HC. Quality of life in surgically palliated complex congenital heart disease. Arch Dis Child. 1994; 70(5):382-386. [PubMed: 7517133]

34. Varni JW, Seid M, Kurtin PS. PedsQL 4.0: reliability and validity of the Pediatric Quality of Life Inventory version 4.0 generic core scales in healthy and patient populations. Med Care. 2001; 39(8):800-812. [PubMed: 11468499]

35. Jackson AC, Frydenberg E, Liang RP, Higgins RO, Murphy BM. Familial impact and coping with child heart disease: a systematic review. Pediatr Cardiol. 2015; 36(4):695-712. [PubMed: 25618163]

36. Brosig CL, Whitstone BN, Frommelt MA, Frisbee SJ, Leuthner SR. Psychological distress in parents of children with severe congenital heart disease: the impact of prenatal versus postnatal diagnosis. J Perinatol. 2007; 27(11):687-692. [PubMed: 17717519]

37. Browne G, Bramston P. Stress and the quality of life in the parents of young people with intellectual disabilities. J Psychiatr Ment Health Nurs. 1998; 5(5):415-421. [PubMed: 10067490]

38. Rychik J, Donaghue DD, Levy S, et al. Maternal psychological stress after prenatal diagnosis of congenital heart disease. J Pediatr. 2013; 162(2):302-307. e301. [PubMed: 22974576]

39. Kolaitis GA, Meentken MG, Utens E. Mental health problems in parents of children with congenital heart disease. Front Pediatr. 2017; 5:102. [PubMed: 28534022]

40. Arya B, Glickstein JS, Levasseur SM, Williams IA. Parents of children with congenital heart disease prefer more information than cardiologists provide. Congenit Heart Dis. 2013; 8(1):78-85. [PubMed: 22891764]

41. Uzark K, Jones K. Parenting stress and children with heart disease. J Pediatr Health Care. 2003; 17(4):163-168. [PubMed: 12847425] 
42. Teixeira FM, Coelho RM, Proenca C, et al. Quality of life experienced by adolescents and young adults with congenital heart disease. Pediatr Cardiol. 2011; 32(8):1132-1138. [PubMed: 21710181]

43. Goldbeck L, Melches J. Quality of life in families of children with congenital heart disease. Qual Life Res. 2005; 14(8):1915-1924. [PubMed: 16155778]

44. Lawoko S, Soares JJ. Quality of life among parents of children with congenital heart disease, parents of children with other diseases and parents of healthy children. Qual Life Res. 2003; 12(6): 655-666. [PubMed: 14516175] 


\section{Table 1}

Subject demographics, CMR, and CPET variables

\begin{tabular}{llll}
\hline & VPS & TOF & P values \\
\hline Number of subjects & 19 & 12 & \\
Age at testing & $15.8 \pm 3.5$ & $15.6 \pm 2.4$ & $0.70^{*}$ \\
Males (\%) & 63 & 67 & 0.72 \\
Caucasian (\%) & 75 & 94 & 0.14 \\
Years of exposure to PI & $14 \pm 4.1$ & $14 \pm 2.7$ & $1.0^{*}$ \\
CMR & & & \\
Pulmonary regurgitant fraction $(\%)$ & $17.9 \pm 11.8$ & $25.9 \pm 11.3$ & $0.05^{*}$ \\
RV end-diastolic volume $\left(m_{1} / m_{2}\right)$ & $94.1 \pm 29.9$ & $110.4 \pm 24.6$ & 0.14 \\
RV end-systolic volume $\left(m_{1} / m_{2}\right)$ & $34.9 \pm 16$ & $44.8 \pm 18.9$ & 0.15 \\
RV ejection fraction $(\%)$ & $64.7 \pm 7$ & $60.9 \pm 8.8$ & 0.21 \\
CPET & & & \\
Indexed oxygen pulse $\left[\left(\mathrm{ml} / \mathrm{O}_{2}\right) /\left(\right.\right.$ beat $\left.\left./ \mathrm{m}^{2}\right)\right]$ & $6.5 \pm 1.7$ & $6.1 \pm 1.5$ & 0.57 \\
Percent-predicted $\mathrm{mV} \mathrm{O}_{2}$ & $82 \pm 10$ & $86 \pm 6$ & 0.19 \\
\hline
\end{tabular}

Subjects recruited to the VPS study were of similar age as those recruited as part of the TOF study. By study design, subjects were matched by duration and severity of pulmonary insufficiency 


\section{Table 2}

Cardiac interventions

\begin{tabular}{lr}
\hline & $\begin{array}{r}\text { TOF } \\
\mathbf{n = 1 2}\end{array}$ \\
\hline Cardiac surgeries $^{a}$ & 12 \\
Complete TOF repair & 12 \\
Palliative procedure (BT shunt) & 3 \\
Reoperation for residual lesion & 4 \\
Cardiac catheterization procedures ${ }^{a}$ & \\
Diagnostic catheterizations & \\
Preoperative & 10 \\
Postoperative & 10 \\
Interventional catheterizations & 3 \\
\hline
\end{tabular}

$B T$ Blalock-Taussig

$a_{\text {Total number of procedures in group }}$ 


\section{을 \\ 졸}

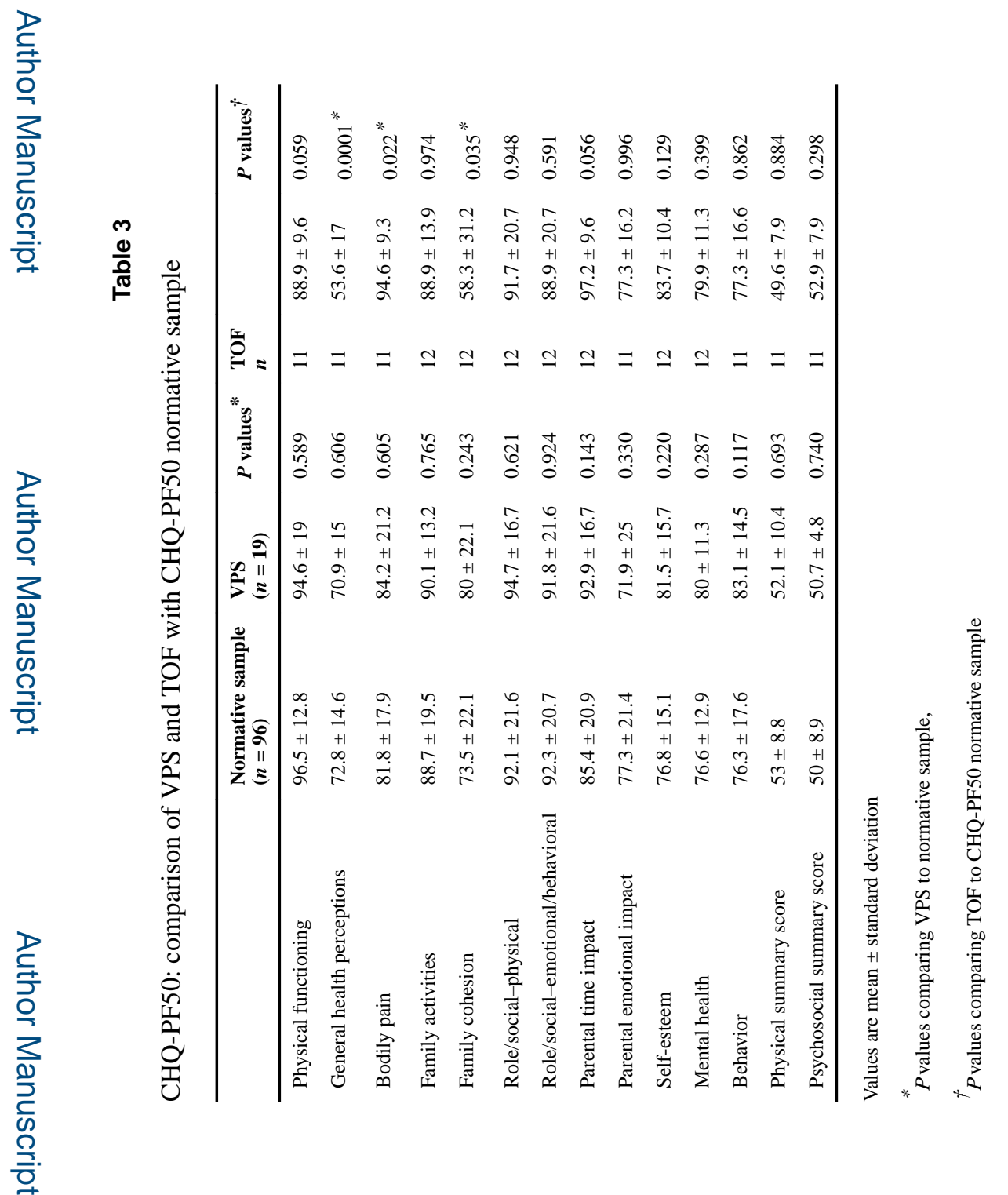

Pediatr Cardiol. Author manuscript; available in PMC 2018 December 01. 


\section{로을}

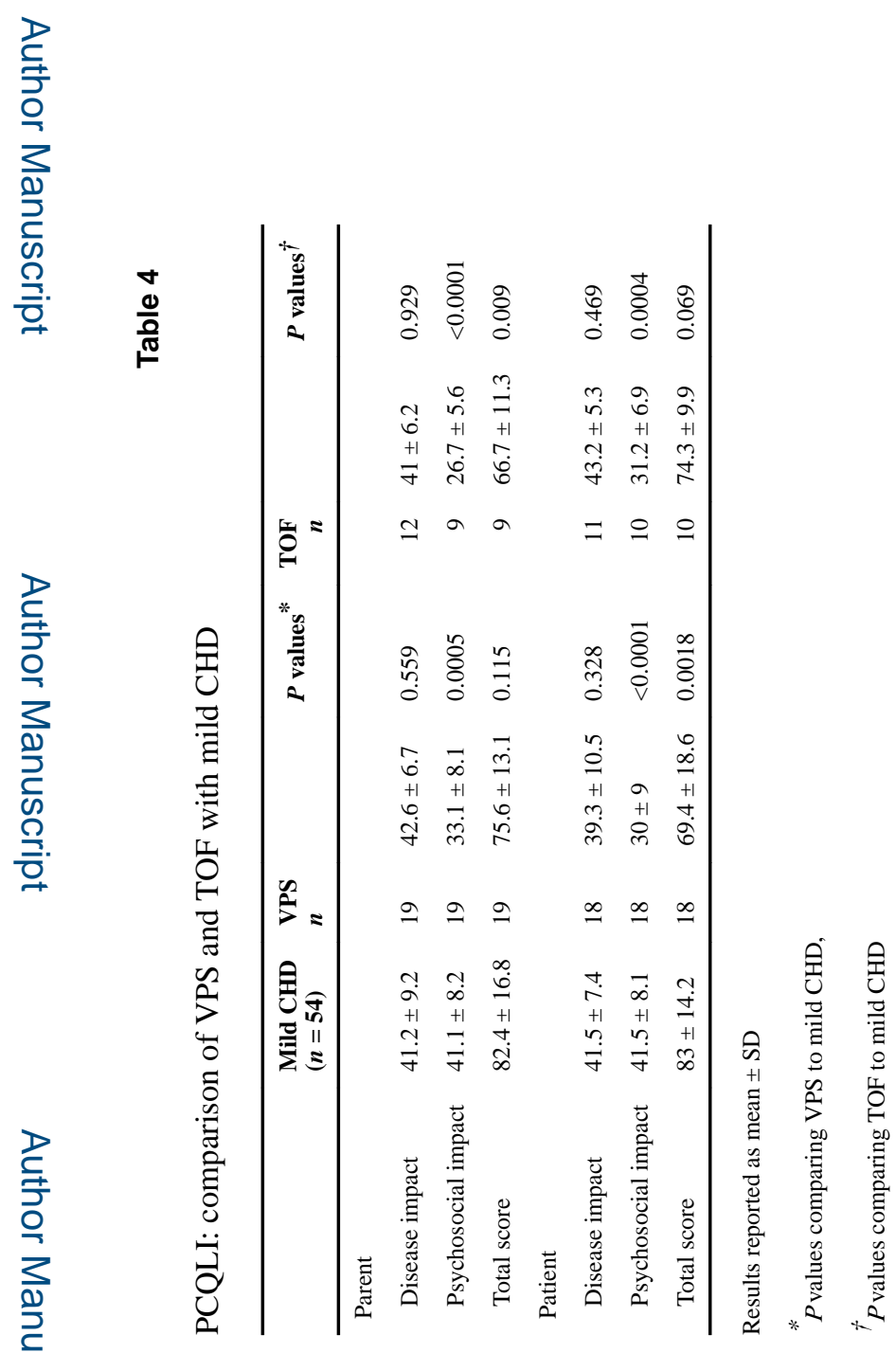

Pediatr Cardiol. Author manuscript; available in PMC 2018 December 01. 


\section{로을}

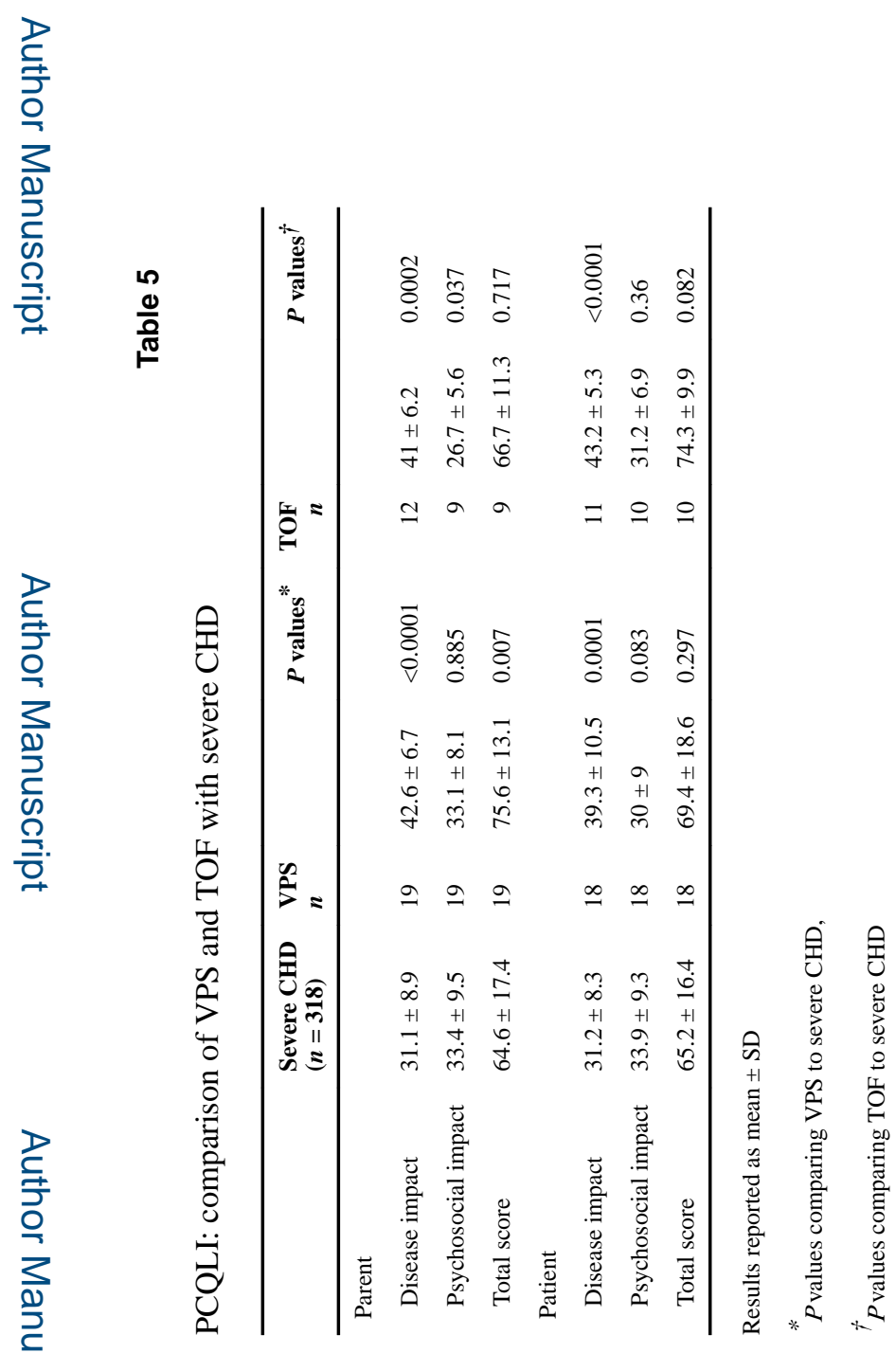

Pediatr Cardiol. Author manuscript; available in PMC 2018 December 01. 
Table 6

Summary of CHQ-PF50: comparison of VPS with TOF

\begin{tabular}{llll}
\hline Variables & VPS & TOF & $\boldsymbol{P}$ values \\
\hline Global health & $93(85-100)$ & $85(60-100)$ & 0.180 \\
Physical functioning & $100(100)$ & $89(78-100)$ & $0.010^{*}$ \\
Role/social-emotional/behavioral & $100(100)$ & $100(83-100)$ & 0.476 \\
Role/social-physical & $100(100)$ & $100(100)$ & 0.626 \\
general health perceptions & $75(58-83)$ & $58(43-67)$ & $0.016^{*}$ \\
Bodily pain/discomfort & $100(70-100)$ & $100(80-100)$ & 0.221 \\
Family activities & $96(79-100)$ & $94(81-100)$ & 0.627 \\
Family cohesion & $85(60-100)$ & $60(30-85)$ & $0.048 *$ \\
Parental time impact & $100(89-100)$ & $100(100)$ & 0.251 \\
Parental emotional impact & $75(50-100)$ & $75(58-92)$ & 0.695 \\
Self-esteem & $83(75-92)$ & $83(77-90)$ & 0.932 \\
Mental health & $80(75-90)$ & $80(70-89)$ & 0.902 \\
Behavior & $85(71-96)$ & $73(64-93)$ & 0.298 \\
Global behavior & $85(60-100)$ & $85(60-85)$ & 0.169 \\
Changes in health & Same 79\% & Same 64\% & 0.824 \\
& Better 21\% & Better 27\% & \\
Physical summary score & Worse 0\% & Worse 9\% & \\
Psychosocial summary score & $55(50-57)$ & $51(47-54)$ & 0.169 \\
\hline & $52(48-55)$ & $53(46-59)$ & 0.561 \\
\hline
\end{tabular}

Results reported as median (interquartile range) 
Table 7

CHQ-CF87 and PCQLI: comparison of VPS with TOF

\begin{tabular}{|c|c|c|c|}
\hline Subscales (abbreviation) & VPS & TOF & $P$ values \\
\hline Global health (GGH) & $85(60-100)$ & $85(60-100)$ & 0.842 \\
\hline Physical functioning (PF) & $100(93-100)$ & $96(96-100)$ & 0.913 \\
\hline Role/social limitations: emotional (RE) & $100(78-100)$ & $100(100)$ & 0.057 \\
\hline Role/social limitations: behavioral (RB) & $100(100)$ & $100(100)$ & 0.311 \\
\hline Role/social limitations: physical (RP) & $100(100)$ & $100(100)$ & 0.39 \\
\hline General health perceptions (GH) & $70(53-82)$ & $69(61-74)$ & 0.730 \\
\hline Bodily pain/discomfort (BP) & $80(60-100)$ & $80(70-90)$ & 0.778 \\
\hline Family activities (FAs) & $100(63-100)$ & $96(90-100)$ & 0.640 \\
\hline Family cohesion (FC) & $85(60-100)$ & $60(30-85)$ & 0.066 \\
\hline Self-esteem (SE) & $77(68-96)$ & $79(73-85)$ & 0.541 \\
\hline Mental health (MH) & $72(63-80)$ & $82(73-88)$ & $0.036^{*}$ \\
\hline Behavior (BE) & $82(68-88)$ & $76(73-88)$ & 0.846 \\
\hline Global behavior (GBE) & $85(60-100)$ & $85(60-100)$ & 0.909 \\
\hline \multirow[t]{3}{*}{ Changes in health $(\mathrm{CH})$} & Same $58 \%$ & Same $75 \%$ & 0.633 \\
\hline & Better $37 \%$ & Better $25 \%$ & \\
\hline & Worse $5 \%$ & Worse $0 \%$ & \\
\hline \multicolumn{4}{|l|}{ PCQLI } \\
\hline \multicolumn{4}{|l|}{ Patient } \\
\hline Disease impact & $41(35-47)$ & $44(39-47)$ & 0.380 \\
\hline Psychosocial impact & $31(29-33)$ & $32(28-35)$ & 0.648 \\
\hline Total patient score & $73(67-79)$ & $78(66-81)$ & 0.486 \\
\hline \multicolumn{4}{|l|}{ Parent } \\
\hline Disease impact & $44(39-49)$ & $40(38-46)$ & 0.360 \\
\hline Psychosocial impact & $31(28-38)$ & $29(24-29)$ & 0.126 \\
\hline Total parent score & $76(64-83)$ & $69(61-76)$ & 0.115 \\
\hline
\end{tabular}

Pediatr Cardiol. Author manuscript; available in PMC 2018 December 01. 